\title{
A case of hashimoto's encephalopathy in a patient with lithium toxicity
}

\begin{abstract}
Hashimoto's encephalopathy is a rare neurological disorder of unknown etiology. Prevalence of $\mathrm{HE}$ is $2.1 / 100,000$. We presented a case of a middle aged male with bipolar disorder on treatment (Lithium carbonate) since 30 years, admitted with altered behavior for 2 weeks and high Lithium levels. His EEG showed generalized epileptiform discharges. MRI brain revealed significant frontal cortical atrophy. CSF studies were normal. Further workup showed a high TSH level and positive anti-TPO (anti thyroid peroxidase) antibodies. Based on clinical picture, raised antibodies and no discernible cause, diagnosis of probable Hashimoto's encephalopathy was made .He received pulse of methylprednisolone for five days and his symptoms improved dramatically. To our knowledge this is the first case of hashimoto's encephalopathy in a patient of bipolar disorder with lithium toxicity .Our case report highlights the importance of diagnosing a rare neurological syndrome in a complex clinical scenario.
\end{abstract}

Keywords: hashimoto's encephalopathy, lithium toxicity, rare
Volume 6 Issue 5 - 2017

\section{Saba Zaidi, Bashir Soomro}

Neurology Consultant at Ziauddin University Hospital, Pakistan

Correspondence: Bashir Soomro, Neurology Consultant at Ziauddin University Hospital, Pakistan, Tel 0300-822-3647, Email basoomro@gmail.com

Received: March 05, 2017 | Published: May 05, 2017

\section{Introduction}

Hashimoto's encephalopathy (HE) is an uncommon neurological syndrome associated with Hashimoto's thyroiditis, first reported by Brain et al in 1966. The disorder occurs more frequently between age 44 to 46 years, with a female to male ratio of $4: 1$. The clinical presentation may involve relapsing remitting course and include stroke like episodes, cognitive decline, neuropsychiatric symptoms, seizures and myoclonus. Thyroid status may vary from hyperthyroid, euthyroid to hypothyroid. Treatment with corticosteroids is almost always successful. Other forms of immunomodulation, such as intravenous immune-globulin and plasma exchange, may also be effective. We presented a case of hashimoto's encephalopathy in a patient of known bipolar disorder with lithium toxicity.

\section{Case report}

We presented a case of 55 years old businessman, known case of Hypertension and Bipolar disorder since 30 years. He was admitted thru outpatient department with irrelevant talking for 2 weeks. His family was concerned for the recent changes in the patient's behaviour as he was not recognizing, passing stool in the bedroom, extremely irritable and agitated. He also left home for 2 days and was found unresponsive outside shopping mall. During these two weeks patient has been unpredictable, responding to internal stimuli and smiling. In hospital whenever asked about his health he usually replies: fuck you. He had history of tremors of both hands for the past 6 months. There was a history of stimulant use in the past as well. He was under treatment for bipolar disorder receiving Lithium $300 \mathrm{mg}$ thrice daily, Carbamazepine $200 \mathrm{mg}$ twice daily for the past 30 years and for hypertension on Lisinopril $5 \mathrm{mg}$ daily. On examination, he was a middle-aged male, lying on bed, conscious, aggressive, not following commands, there was significant palilalia, cranial nerves were grossly intact. Motor examination showed significant increase in tone in all four limbs, no focal deficit, reflexes were normal. Plantars were flexor. No signs of meningeal irritation. No nystagmus or myoclonus appreciated. His baseline workup revealed $\mathrm{Hb}$ of 9.6 $\mathrm{gm} / \mathrm{dl}$ (Normocytic normochromic) deranged renal parameters (urea: $150 \mathrm{mg} / \mathrm{dl}$, Creatinine: $3.44 \mathrm{mg} / \mathrm{dl}$ ), TSH of $6 \mathrm{Miu} / \mathrm{L}(0.4-4)$ Lithium levels of $3.9 \mathrm{mmol} / \mathrm{L}(0.4-2 \mathrm{mmol} / \mathrm{L})$. His lithium was stopped and intravenous hydration was started. EEG and MRI Brain were done. EEG showed generalized epileptiform discharges. MRI brain showed frontal cortical atrophy, no post contrast enhancement. CSF studies were normal. HIV and VDRL serologies were negative. Patient was started on Acyclovir and Sodium valproate for epileptiform discharges. He received Haloperidol and later, kept on Quitiapine (25 mg twice a day) for agitation. During the three days of hospital stay there was no improvement in his condition. He remained encephalopathic. Repeat Lithium levels of $0.8 \mathrm{mmol} / \mathrm{L}(0.4-2)$. His HSV PCR came out as negative. His workup was sent for autoimmune encephalitis which was all negative. Thyroid antibodies were also sent with the probability of hashimoto's encephalopathy. His anti TPO (anti-thyroid peroxidase) came out to be positive and patient was given pulse of methylprednisolone for 5 days. His symptoms improved dramatically and he was discharged after 7 days, on tapering doses of steroids. Repeat Urea $91 \mathrm{mg} / \mathrm{dl}$, Creatinine $1.88 \mathrm{mg} / \mathrm{dl}$, Hemoglobin of $10 \mathrm{mg} /$ dl. Ultrasound kidneys showed bilateral renal parenchymal disease (Figure 1).

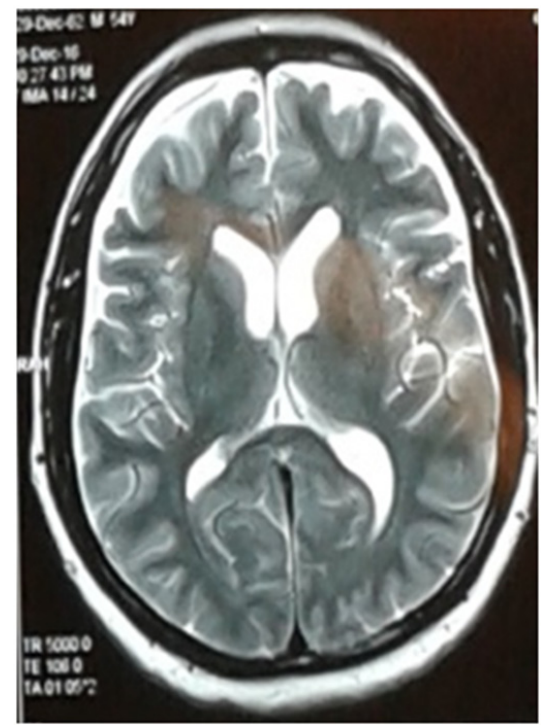

Figure I T2 Weighted image showed significant frontal cortical atrophy. 


\section{Discussion}

Hashimotos encephalopathy is a rare neurological disorder. It was described by Brain and co-workers in $1966 .{ }^{1}$ Prevalence of HE is $2.1 / 100,000$. The disorder occurs more frequently between ages 44 to 46 years, with a female-to-male ratio of $4: 1 .^{2}$ Two major patterns of presentation were described: in $25 \%$ of cases stroke-like pattern of multiple recurrent episodes of focal neurologic deficits and the remaining $75 \%$ present with a diffuse progressive pattern of cognitive decline with dementia, confusion and hallucinations. ${ }^{1-3}$

Other rare presentations include: Paranoid delusions, psychosis, visual hallucinations in $25 \%$ to $36 \%$ of patients. ${ }^{2}$ Myoclonus or tremor is seen in up to $38 \%$ of patients. Focal or generalized tonic-clonic seizures have been reported in two-third of cases, and $12 \%$ may present with status epilepticus. ${ }^{2,3}$ Thyroid status in hashimoto's encephalopathy may vary from hypothyroid, euthyroid to hyperthyroid.

Literature review revealed that among patients with $\mathrm{HE}, 23 \%$ to $35 \%$ of patients had subclinical hypothyroidism, $17 \%$ to $20 \%$ had hypothyroidism, $7 \%$ had hyperthyroidism and $18 \%$ to $45 \%$ were euthyroid. Our described case was hypothyroid.

Diagnosis of HE is usually made by excluding other toxic, metabolic and infectious causes of encephalopathy on MRI brain, CSF studies and EEG. Although the CSF analysis was performed in our patient was normal, a lymphocytic pleocytosis has been found in $14 \%$ of reported patients of HE. An elevated protein concentration occurs in $78 \%$ of patients. The blood glucose concentration is usually normal. ${ }^{4}$ EEG abnormalities were seen in $90 \%$ to $98 \%$ of patients, usually a nonspecific slow background activity. Focal spikes or sharp waves and transient epileptic activity are less common. ${ }^{5}$ In a review of 82 patients with HE, brain computed tomography or MRI showed abnormalities in $49 \%$ such as cerebral atrophy, focal cortical abnormality, diffuse subcortical abnormality and nonspecific subcortical focal white matter abnormality. ${ }^{6}$

In the context of the typical clinical spectrum as mentioned, high titers of antithyroid antibodies, in particular antithyroid peroxidase antibodies, are diagnostic. The diagnosis of HE should be considered even if Hashimoto's thyroiditis is not known in patients with euthyroid states but with high serum and/or CSF titers of antithyroid antibodies .Treatment with corticosteroids is usually successful, although relapse may occur if this treatment is stopped abruptly. Other therapeutic strategies should be tried: immunosuppressive therapy (azathioprine or cyclophosphamide) and recently proposed in some cases are plasmapharesis or intravenous immunoglobulins. Our patient responded well to corticosteroid pulse therapy. ${ }^{7,8}$

Our patient was admitted with the impression of Lithium toxicity as his levels were high. Literature review of 52 cases of Lithium neurotoxicity revealed that it usually presents with pyramidal, extrapyramidal and cerebellar signs. Some of the important findings were ataxia, myoclonus, dysarthria, convulsions and tremors. Some rare presentations include, neuroleptic malignant syndrome, serotonin syndrome and pseudotumor cerebri. Other features include intention tremor, rigidity, confusion, disorientation, urinary retention, and increased creatine kinase levels. ${ }^{9}$ None of these presentations, mimics our case of severe encephalopathy, this made us think for an alternate diagnosis in the presence of deranged metabolic parameters and high Lithium levels.

\section{Acknowledgments}

Dr Imran Chaudary, Psychiatrist at Ziauddin university Clifton Campus.

Dr Fasiha Sohail. Assistant Professor Internal Medicine, Ziauddin university Clifton Campus.

\section{Conflicts of interest}

None.

Funding

None.

\section{References}

1. Chong JY, Rowland LP, Utiger RD. Hashimoto encephalopathy: syndrome or myth? Arch Neurol. 2003;60(2):164-171.

2. Ferracci F, Carnevale. The neurological disorder associated with thyroid autoimmunity. AJ Neurol. 2006;253(8):975-984.

3. Kothbauer-Margreiter I, Sturzenegger M, Komor J, et al. Encephalopathy associated with Hashimoto thyroiditis: diagnosis and treatment. $J$ Neurol. 1996;243(8):585-593.

4. Mocellin R, Walterfang M, Velakoulis D. Hashimoto's encephalopathy: epidemiology, pathogenesis and management. CNS Drugs. 2007;21(10):799-811.

5. Ferracci F, Carnevale A. The neurological disorder associated with thyroid autoimmunity. J Neurol. 2006;253(8):975-984.

6. Peschen-Rosin R, Schabet M, Dichgans J. Manifestation of Hashimoto's encephalopathy years before onset of thyroid disease. Eur Neurol. 1999;41(2):79-84.

7. Ferracci F, Bertiato G, Moretto G. Hashimoto's encephalopathy: epidemiologic data and pathogenetic considerations. J Neurol Sci. 2004;217(2):165-168.

8. Kothbauer-Margreiter I, Sturzenegger M, Komor J, et al. Encephalopathy associated with Hashimoto thyroiditis: diagnosis and treatment. J Neurol . 1996;243(8):585-593.

9. Netto I, Phutane H. Reversible lithium toxicity: Review of literature. Prim Care Companion CNS Disord. 2012; 14(1). 